\title{
Hemoglobinopathy Toms River
}

INSERM

\section{Source}

INSERM. (1999). Orphanet: an online rare disease and orphan drug data base.

Hemoglobinopathy Toms River. ORPHA:280615

Hemog lobinopathy Toms River is a rare, genetic hemoglobinopathy disorder, due to a defect in the gama subunit of the fetal hemoglobin, characterized by neonatal cyanosis, low hemoglobin oxygen saturation levels without arterial hypoxemia, moderate anemia and reticulocytosis, not associated with heart or lung disease. Symptoms progressively subside within the first months of life. 